\title{
La imagen comercial y su influencia socio económica empresarial de los pequeños negocios del cantón Sucre. Ecuador
}

\section{The commercial image and its socio-economic influence of the small businesses of the canton Sucre. Ecuador}

Frank Á Lemoine Quintero

Universidad Laica Eloy Alfaro de Manabí, Ecuador

Gema V Carvajal Zambrano

Universidad Laica Eloy Alfaro de Manabí, Ecuador

Maryury Alexandra Zamora Cusme

Escuela Superior Politécnica Agropecuaria de Manabi "Manuel Félix López", Ecuador

Graciela Castellanos Pallerols

Universidad de Oriente, Cuba

Autor para correspondencia: fangel64@gmail.com, vivicarvajalzambrano@gmail.com, graciela@ri.uo.edu.cu, maryuryzamora01@hotmail.com

Fecha de recepción: 28 de Agosto 2017 - Fecha de aceptación: 15 de Enero de 2018

Resumen: La investigación se sustenta en contribuir al desarrollo sustentable y socioeconómico empresarial de los pequeños negocios con el objetivo de plasmar una filosofía de servicio que le propicie el cumplimiento de normas empresariales. Los objetivos propuestos están en diagnosticar el entorno empresarial de la parroquia para medir su impacto en las comunidades y evaluar las microempresas a partir de su imagen comercial para definir sus sustentabilidad económica. El método estadístico descriptivo permitió realizar un levantamiento como línea base de la investigación, donde las tiendas barriales represento el 35,7\% del total de locales, seguido de los comedores o restaurantes con un 12,9\% de representatividad. El estudio del entorno empresarial permitió evaluar el comportamiento de las fuerzas actuantes a nivel de actividad laboral, nivel de instrucción y otras variables consideradas para el estudio. Se analizó la imagen comercial de los negocios donde $67,3 \%$ reconoce que la imagen tiene los elementos de la imagen empresarial y solo un $37,2 \%$ desconoce los elementos de la imagen comercial. Como resultado se logra que el 50\% de los negocios y locales reducen la informalidad laboral garantizando el cumplimiento de los derechos laborales y que el $70 \%$ de los negocios desarrollaron estrategias y técnicas que incrementaron las ventas.

Palabras claves: imagen comercial; diagnóstico; entorno; sustentable; impacto

Abstract: The research is based on contributing to the sustainable and socioeconomic development of small businesses with the objective of forming a philosophy of service that promotes compliance with business rules. The proposed objectives are to diagnose the business environment of the parish to measure their impact on communities and to evaluate microenterprises based on their commercial image to define their economic sustainability. The descriptive statistical method allowed a survey to be carried out as a baseline of the investigation, 
where the neighborhood stores represented $35.7 \%$ of the total of premises, followed by the dining rooms or restaurants with a $12.9 \%$ representativeness. The study of the business environment made it possible to evaluate the behavior of the forces acting at the level of work activity, level of education and other variables considered for the study. The commercial image of the businesses was analyzed, where $67.3 \%$ recognize that the image has the elements of the corporate image and only $37.2 \%$ ignore the elements of the commercial image. As a result, $50 \%$ of businesses and local businesses reduce labor informality by ensuring compliance with labor rights and that $70 \%$ of businesses developed strategies and techniques that increased sales.

Key words: commercial image; diagnosis; environment; sustainable; impact

\section{Introducción}

Las microempresas en el Ecuador tiene la capacidad de contribuir al desarrollo socioeconómico del país, además de acelerar la generación de empleos e incrementar el ingreso familiar por la particularidad de que estas tributan al emprendimiento familiar como soporte económico. Tiene la ventaja de aprovechar los recursos internos que existen en las áreas urbanas y rurales como oportunidad para posicionarse en el mercado.

Las nuevas políticas generada hoy en día en el país han generado que la economía popular esta direccionada a actividades económicas que contribuyen a diferentes sectores económicos del país y prácticas sociales desarrolladas por los sectores populares con miras a garantizar, a través de la utilización de su propia fuerza de trabajo y de los recursos disponibles, la satisfacción de las necesidades básicas, tanto materiales como inmateriales.

El Consejo Empresarial para el Desarrollo Sostenible del Ecuador (CEMDES) estableció el Plan Nacional de Desarrollo y su continuidad con el Plan Nacional para el Buen Vivir, como carta de navegación del país. Enfatizó que dentro de los objetivos de desarrollo del Plan se incluye la dinámica privada, a través de sus correspondientes estrategias, que a su vez este permite impulsar actividades empresariales sustentables a nivel empresarial.

Fundamentamos en el Artículo 284 de la Constitución de la República establece los objetivos de la política económica, entre los que se incluye incentivar la producción nacional, la productividad y competitividad sistémicas, la acumulación del conocimiento científico y tecnológico, la inserción estratégica en la economía mundial y las actividades productivas complementarias en la integración regional, según el código orgánico de la producción del Ecuador, 2016

Como también en el Art. 22.- Medidas específicas.- El Consejo Sectorial de la Producción establecerá políticas de fomento para la economía popular, solidaria y comunitaria, así como de acceso democrático a los factores de producción, sin perjuicio de las competencias de los Gobiernos Autónomos Descentralizados y de la institucionalidad específica que se cree para el desarrollo integral de este sector, de acuerdo a lo que regule la Ley de esta materia. Adicionalmente, para fomentar y fortalecer la economía popular, solidaria y comunitaria, el Consejo Sectorial de la Producción ejecutará las siguientes acciones. 
Relevando la importancia que pueden alcanzar los negocios en el crecimiento sustentable de una comunidad o localidad y así fomentar programas y proyectos para el desarrollo y avance de la producción nacional, regional, provincial y local, además de apoyar y consolidar el modelo socio productivo comunitario para lo cual elaborará programas y proyectos con financiamiento público para: recuperación, apoyo y transferencia tecnológica, investigación, capacitación y mecanismos comercialización y de compras públicas (Segundo Suplemento del Registro Oficial 056, 12-VIII-2013)

En el Ecuador en el último año ha existido un importante incremento de las micro, pequeñas y medianas empresas al pasar del $38 \%$ registrado en el 2011 al $41 \%$ en el presente año lectivo, precisó Ricardo Zambrano, subsecretario de Mipymes y Artesanías del Ministerio de Industrias y Productividad (Mipro).

La parroquia de Leónidas Plaza ubicada en el cantón Sucre en la provincia de Manabí cuenta con 36 barrios y comunidades anexas con una población aproximada de 22.000 habitantes, tiene un crecimiento poblacional y comercial bien representativo en el cantón y considerada como el impulsor de las actividades comerciales de la ciudad de Bahía de Caráquez.

Después del acontecimiento en Ecuador del 16 de Abril del año 2016, hubo una gran afectación en la ciudad de Bahía de Caráquez, generando un incremento de negocios en la parroquia de Leonidas Plaza de un 102\%, el cual hizo que la competencia incrementara debido a que los negocios de la ciudad afectada se ubicaran en dicha parroquia.

La existencia de locales que les brinda servicios a pobladores y visitantes nacionales e internacionales que asisten a la parroquia no guardan una relación con su razón social y su imagen comercial por lo que esto afecta los servicios que brindan los mismos.

Entre los servicios más representativos están las tiendas barriales, los pequeños comedores y comidas rápidas, esto debido a la afluencia tanto comercial por su ubicación geográfica como número de entidades públicas ubicadas representativas en la parroquia tales como hospital, Universidad y Consejo de la Judicatura entre otras de menor representatividad.

Para la realización de este proyecto incurrió la investigación de necesidades existente en el entorno, la cual se pudo encontrar falencias en las estructuras que caracterizan a los locales comerciales de nuestra zona y falencia en los servicios que prestan, tal es el caso de que muchos locales cuentan con la misma apariencia desde sus inicios y sus propietarios desconocen de técnicas y estrategias de ventas.

El deficiente servicio de los locales y negocios existente en la parroquia Leónidas Plazas afecta hoy en día a la sustentabilidad económica de su población lo que resulta una preocupación en investigadores y especialista en el tema por el impacto que pude generar tanto de índole de desempleo como afectaciones económicas en las familia de la zona.

Las principales causas están dada por el déficit de conocimiento de técnicas de ventas de los dueños o propietarios de los negocios, el poco conocimiento del Marketing Empresarial Sostenible que permita su permanencia y sustentabilidad en el mercado, el deterioro de imágenes 
corporativas en los pequeños negocios que no lo identifican con los servicios que prestan que a su vez estos generan efectos que atentan con la permanencia de los mismos.

\section{Metodología}

Pintado Blanco \& Sánchez Herrera, 2013) dice que la imagen corporativa es uno de esos escasos factores dentro de las organizaciones, que todo el mundo considera fundamentales y, que sin embargo, no siempre se cuidan como merecen.

Se puede definir como una evocación o representación mental que conforma cada individuo, formada por sus atributos referentes a la compañía; cada uno de esos atributos puede variar, y puede coincidir o no con la combinación de atributos ideal de dicho individuo.

Según (Sánchez y Pintado, 2009) conseguir una imagen positiva, es necesario proyectarla cuando la identidad de la empresa es clara y esté definida. Además hay que tener en cuenta que es un proceso cotidiano, por lo cual la formación de la imagen corporativa representa "cualquier acto de la empresa puede conllevar a la aparición de una imagen concreta, por lo que hay que estar permanentemente alerta, con el fin de que no haya fisuras que permitan que el público se lleve una imagen negativa"

Para Bort (2004), la imagen corporativa desde cualquier punto de vista debe cumplir dos requisitos básicos, los cuales son uniformidad y presencia. Según Ana Jiménez (2007) argumenta que "la imagen corporativa es resultado de un complejo proceso desarrollado en la mente del individuo en el que intervienen multitud de factores, internos y externos al sujeto. Además, este proceso se encuentra condicionado por el uso de diversas fuentes, que ofrecen información sobre la empresa, que, de diferente tipo, es cada en distinta cantidad y con diferente nivel de calidad y objetividad".

Estructurada en tres etapas: Etapa 1: Diagnóstico, Etapa 2: Análisis del entorno empresarial y en la Etapa 3: Impacto social de la imagen empresarial. Cada una de esta etapas diseñadas con un análisis objetivo de la situación problemica del entorno que aporten a la investigación, al desarrollo de los negocios con técnicas, métodos y herramientas de marketing que fortalezca la actividad empresarial de trabajadores y empresarios del sector de servicio.

Se empleó el método de observación que consistió en saber seleccionar aquello que queremos analizar. Por lo que se realizó una observación partiendo de comportamientos en los locales o negocios, de quejas y anomalías de usuarios o clientes y también la pérdida de identidad cultural en la zona deteriorando la imagen comercial de los negocios existente.

Se aplicó además el método estadístico descriptivo que tuvo como objetivo la descripción precisa del evento de estudio. Este tipo de investigación se asocia al diagnóstico que se realizara en las comunidades, donde se hace enumeración detallada de las características del evento de estudio según criterios del grupo de experto conformado por especialista de la materia para realizar esta investigación. 
En el caso de la investigación estadística descriptiva, la indagación va dirigida a responder a las preguntas quién, qué, dónde, cuándo, cuántos (Borderleau, 1987). Esta investigación descriptiva trabajara con varios eventos de estudio teniendo presente la variables objeto estudios y del el levantamiento estadístico a nivel poblacional, de negocios o locales y de afluencias de clientes.

Se aplicó una encuesta estructurada con preguntas abiertas y cerradas con la finalidad de identificar las principales deficiencias de la imagen empresarial. Se utilizara el software estadístico SPSS 21.00 para procesar la encuesta y realizar el un análisis de fiabilidad. Se utilizara el software REDATAM para definir estudio del entorno empresarial de la localidad. A través del software Publisher se diseñan trípticos para diferentes actividades de publicitarias.

\section{Resultados}

La economía del Cantón se deriva en $37.47 \%$ se dedica al sector terciario donde resalta las actividades de comercio al por mayor y menor, alojamiento, la parte turística, enseñanza, transporte, haciendo este sector en su conjunto el más fuerte del Cantón y basándose su fuerza económica en lo urbano. El sector primario, es la segunda fuerza económica con un 34.98\% basándose a la actividades pecuarias, de agricultura, selvicultura y pesca, teniendo su fuerte en el área rural. Por consiguiente el sector secundario es el menos fuerte en la parte económica del Cantón Sucre con tan solo el 10.67\%, basando este rubro en las empresas de construcción y en las industrias manufactureras. El sector económico no declarado con $9.57 \%$ y por último el trabajador nuevo que ocupa el 7.31\% (Redatam, 2010).

Esto significa que el Cantón Sucre posee su economía en comercio y turismo como fuentes principales, seguido de la agricultura y a fines, su gente trabaja por alcanzar desarrollo económico sostenible en las diferentes actividades, el Cantón se lo conoce como rural por su posicionamiento. Existen actividades que van a ir creciendo y van a tener el lugar y espacio en la economía pero esto a medida que se regulen muchos factores.

El sector comercial y de servicios en el Cantón Sucre genera aproximadamente el 37\% de ingresos por sus diversos lugares turísticos, infraestructura, hospedaje, restaurantes, centros nocturnos, comercio, transporte y más, que ayuda a la parte del desarrollo del Cantón se debe tener presente los impactos económicos, socio-culturales y ambientales los cuales considerar y respetar para mantener el turismo activo y continuar con las actividades principales del Cantón.

La parroquia de Leónidas Plaza está conformada por 33 comunidades o barrios en la zona urbana que representa un $46 \%$ del total de las comunidades de la parroquia Leónidas Plaza existen 33 comunidades y Bahía de Caráquez existen 10 comunidades por lo que en la zona rural de ambas parroquias existen un total de 50 comunidades, barrios o recintos que representa un $54 \%$ del total. El estudio se realiza en la zona urbana de Leónidas Plazas por el número de locales o negocios existente según resultados de la técnica de muestreo realizada.

Se utilizaron dos instrumento, un cuestionario y una técnica de observación o muestreo donde se determinó según criterio de experto cinco variables o parámetros a medir, los dentro de ellos está el tipo o denominación del negocio, el área por metros cuadrados, los clientes 
atendidos diarios, el confort, ambiente y tecnología y el tiempo de apertura del negocio o locales existente en la parroquia.

Como resultado del instrumento una gran parte de estos locales carece de una identidad que los identifique, esto hace que desfavorezca el desarrollo económico de la zona, afectando a los ingresos de los dueños de estos negocios y la afluencia de clientes incidiendo en los bajos ingresos de los locales o negocios.

Del muestreo realizado y procesado a través de la herramienta estadística SPSS 21.0 los resultados obtenidos de las variables medidas fueron los siguientes:

\begin{tabular}{|c|c|c|c|c|c|}
\hline & & Frecuencia & Porcentaje & $\begin{array}{c}\text { Porcentaje } \\
\text { válido }\end{array}$ & $\begin{array}{l}\text { Porcentaje } \\
\text { acumulado }\end{array}$ \\
\hline \multirow[t]{8}{*}{ Válidos } & Tienda & 25 & 35,7 & 36,2 & 36,2 \\
\hline & restaurante & 9 & 12,9 & 13,0 & 49,3 \\
\hline & comida rápida & 4 & 5,7 & 5,8 & 55,1 \\
\hline & salón de belleza & 2 & 2,9 & 2,9 & 58,0 \\
\hline & Cyber & 5 & 7,1 & 7,2 & 65,2 \\
\hline & panadería-dulcerías & 7 & 10,0 & 10,1 & 75,4 \\
\hline & Otros & 17 & 24,3 & 24,6 & 100,0 \\
\hline & Total & 69 & 98,6 & 100,0 & \\
\hline Perdidos & Sistema & 1 & 1,4 & & \\
\hline Total & & 70 & 100,0 & & \\
\hline
\end{tabular}

Cuadro 1: Tipo de denominación del negocio.

Fuente: Resultados del SPSS 21.0.

El más representativo fueron las tiendas barriales que represento el 35,7\% seguido de los comedores o restaurantes con un $12,9 \%$ de representatividad en la parroquia y la existencia de panaderías-dulcerías con un $10 \%$, siendo estas las actividades comerciales más distintiva en la comunidad y las que más aportan a los servicios de la parroquia. Denotando que estas actividades comerciales son vitalicia en las comunidades por formar parte elemental de los servicios básicos de la familia en la zona.

En los resultados medidos por el área de los metros cuadrado con la finalidad de determinar la utilidad del espacio en los locales con la finalidad de realizar pronósticos de las ventas en los locales, definir una proyección de los mismos y su rentabilidad, por lo que fue notorio observar que el área por metro cuadrado de los locales o comercios se encuentra en el rango de 9 a más con un $64,3 \%$ de representación, el $22,9 \%$ posee entre 5 a 8 metros cuadrados y un $10 \%$ posee entre 1 a 4 metros cuadrados para realizar sus actividades de comercio o servicios. Por lo que se observa que los locales aun teniendo un espacio amplio como área de venta dependiendo de su actividad comercial los directivos no lo están utilizando debidamente por lo que puede originarle perdidas en sus ventas.

El nivel de cliente atendido fue medido con la finalidad de medir esta variable y definir estrategias funcionales capaces de mejorar el servicio de los negocios en la zona, cuyos resultados fueron los siguientes:

\begin{tabular}{lrrrrr}
\hline & Frecuencia & Porcentaje & $\begin{array}{c}\text { Porcentaje } \\
\text { válido }\end{array}$ & \multicolumn{2}{c}{$\begin{array}{c}\text { Porcentaje } \\
\text { acumulado }\end{array}$} \\
\hline Válidos & 0 a 10 & 8 & 11,4 & 11,6 & 11,6 \\
\hline
\end{tabular}




\begin{tabular}{llrrrr}
\hline & 11 a20 & 21 & 30,0 & 30,4 & 42,0 \\
& 21 a 30 & 13 & 18,6 & 18,8 & 60,9 \\
& 31 a más & 27 & 38,6 & 39,1 & 100,0 \\
& Total & 69 & 98,6 & 100,0 & \\
Perdidos & Sistema & 1 & 1,4 & & \\
Total & & 70 & 100,0 & & \\
\hline
\end{tabular}

Cuadro 2: Clientes atendidos diarios

Fuente: Resultados del SPSS 21.0

De acuerdo a la información obtenida se puede apreciar que diariamente son atendidas más de 31 personas por local o comercio lo que representa el $38,6 \%$, seguido por el $30 \%$ que corresponde a una atención de 11 a 20 usuarios, de 21 a 30 consumidores son atendidos por otros locales o comercios que indican un $18,6 \%$; finalmente un $11,4 \%$ corresponden a la minoría de clientes atendidos diariamente comprendido en el rango de 0 a 10.

De acuerdo al confort, ambiente y tecnología que presentan los negocios y locales del entorno empresarial a medir dio el siguiente resultado:

\begin{tabular}{llrrrr}
\hline & & Frecuencia & Porcentaje & \multicolumn{1}{c}{$\begin{array}{c}\text { Porcentaje } \\
\text { válido }\end{array}$} & $\begin{array}{l}\text { Porcentaje } \\
\text { acumulado }\end{array}$ \\
\hline Válidos & muy bueno & 9 & 12,9 & 13,4 & 13,4 \\
& bueno & 32 & 45,7 & 47,8 & 61,2 \\
& regular & 22 & 31,4 & 32,8 & 94,0 \\
& malo & 4 & 5,7 & 6,0 & 100,0 \\
& Total & 67 & 95,7 & 100,0 & \\
Perdidos & Sistema & 3 & 4,3 & \\
Total & 70 & 100,0 & \\
\hline \multicolumn{5}{c}{ Cuadro 3: Confort, ambiente y tecnología. } \\
& Fuente: Resultados del SPSS 21.0.
\end{tabular}

La representación del 45,7\% considera que los comercios o locales comerciales tienen un confort, ambiente y tecnología bueno; el 31,4\% lo considera como regular; mientras que un $12,9 \%$ piensa que el ambiente es muy bueno y finalmente el 5,7\% considera que es malo el confort que se brinda en estos comercios o locales comerciales.

Sobre el parámetro de tiempo de creada o apertura del negocio es notorio resaltar que el $90 \%$ de los locales comerciales tienen años de tiempo de creación o apertura y solo un $8,6 \%$ tiene meses en esta actividad comercial, por lo que denomina el tiempo que estos negocios tienen en el mercado.

Este estudio aporto al levantamiento de una línea base con la finalidad no solo de medir el comportamiento de los servicios de los locales y negocios, sino también con la finalidad de medir su impacto y crecimiento a partir de las estrategias y técnicas de marketing que se implementaran como herramientas que permitan la sostenibilidad de los mismos en el tiempo.

\section{Análisis del entorno empresarial.}


Con la finalidad de medir el comportamiento de las fuerzas actuantes del entorno empresarial en la zona objeto estudio como reconocimiento y comportamiento del entorno que nos aporte resultados medibles para la toma de decisiones estratégicas y con el propósito de ver cómo estas fuerzas se influyen en los negocios de las comunidades se realiza el análisis del entorno empresarial.

Es importante argumentar que el nivel de instrucción según estadístico del INEC y el software REDATAM en relación a la variable de nivel de instrucción de la población del cantón Sucre se ha establecido que: el 9.53\% no cuenta con ningún nivel de instrucción, el $5.52 \%$ de la población ha realizado por los centros de alfabetización, el $1.15 \%$ obtuvo el nivel preescolar, el 31.33\% tiene instrucción primaria, el $18.31 \%$ su instrucción es secundaria, el $13.25 \%$ cuenta con educación básica, el $7.5 \%$ hasta bachillerato o educación media, el $1.26 \%$ tiene ciclo post bachillerato, el $9.85 \%$ cuenta con instrucción superior, el $0.75 \%$ tiene post grado y el $1.55 \%$ se ignora su nivel de instrucción. Por lo que contamos con una población activa capaz de enfrentar el entorno y emprender algún negocio o pequeña empresa en nuestra localidad.

Otro indicador está reflejado en la profesión u ocupación de nuestro pobladores que según datos del censo 2010 realizado por el INEC, en relación a la profesión y ocupación de los habitantes del cantón Sucre se presentan las siguientes cifras: el $29.62 \%$ son agricultores y trabajadores calificados, el $11.71 \%$ lo constituyen los trabajadores de los servicios y vendedores, el $13.51 \%$ se encuentra dedicado a ocupaciones elementales, el $9.10 \%$ lo conforman los profesionales científicos e intelectuales, el 7.95\%constituyen los oficiales, operarios y artesanos, el $8.49 \%$ trabajan en apoyo administrativo y el $19.62 \%$ restante se dedican a otras actividades. Reflejado esto en los centros Universitarios como guía instructiva en la formación y creación de empresas.

Estudio de diagnóstico y levantamiento de los negocios, empresas u organizaciones existente en el cantón Sucre se refleja un número de empresas existente las cuales están clasificadas o denominadas por su tamaño. Según estas crecen en sus ventas anuales en un 5\% por lo que requiere potencializar personal capacitado en áreas administrativas. Es notable observar en la investigación realizada en el diagnóstico empresarial del entorno que el $48 \%$ responden a microempresas en Bahía, el 21\% representan a las pequeñas empresas y el 17\% solo de medianas empresas siendo estas la de mayores proyecciones en su productividad al mercado exterior y de mejores niveles de crecimiento de sus utilidades. El sector público solo represento el $14 \%$ que se identificaron como las grandes empresas por su estructura organizativa y por el número de trabajadores que elaboran en la misma según se muestra a continuación:

Tabla 1: Denominación de empresas en Bahía de Caráquez.

\begin{tabular}{ccc}
\hline Denominación & Cantidad & Promedio \\
\hline Microempresa & 20 & $48 \%$ \\
Pequeña empresa & 9 & $21 \%$ \\
Mediana empresa & 7 & $17 \%$ \\
Gran empresa & 6 & $14 \%$ \\
& 42 & $100 \%$ \\
\hline
\end{tabular}

\section{Comportamiento del entorno y fuerzas actuantes.}


Es necesario considerar las variables externas, las cuales nos permitían definir el escenario que se espera durante la aplicación y ejecución del plan de mercadotecnia tanto de empresas hoteleras como gastronómicas, como de las pymes. Tradicionalmente, este análisis se concentraba al estudio de las variables no controlables; sin embargo, debido a la evolución de la mercadotecnia, ha sido necesario establecer una nueva estructura de análisis en los estudios de estas fuerzas. Se deben considerar los elementos incontrolables que tienen relación cercana con la empresa o departamento que le afectan de manera directa y que no pueden ser totalmente manejados por el área de mercadotecnia. Los mismos forman parte del ambiente externo, pero por la cercanía, relación e influencia que ejercen en el departamento.

El análisis PEST es una herramienta de gran utilidad para comprender el crecimiento o declive de un mercado, y en consecuencia, la posición, potencial y dirección de un negocio. Es una herramienta de medición de negocios. El PESTE está compuesto por las iniciales de factores Políticos, Económicos, Sociales y Tecnológicos, Éticos o Ecológicos utilizados para evaluar el mercado en el que se encuentra un negocio o unidad, dentro de estas fuerzas a través del software REDATAM se definen las siguientes:

\section{Ámbito socio cultural:}

\section{Etnia}

Etnicidad según datos del INEC (Censo 2010) la población del cantón Sucre en un $58.72 \%$ mestizo, el $35.64 \%$ son montubio, el $3.18 \%$ son indígenas, el $0.75 \%$ son afro ecuatorianos, el $0.68 \%$ son mulatos, el $0.63 \%$ son blancos, el 0.19 son negros y el $0.22 \%$ restante corresponden a miembros de otras etnias.

\section{Población}

De acuerdo a los datos del INEC dado por el software REDATAM basado en el Censo del 2010,la población del Cantón Sucre representa el $4.2 \%$ del total de la Provincia de Manabí; contando con 57.159 habitantes, de los cuales el 63\% habita en las zonas rurales y el $37 \%$ restante reside en las áreas urbanas. La población ha crecido en el período intercensal 1990-2001, a un ritmo del 3.2\% promedio anual; mientras que del período intercensal 2001-2010 ha crecido en un promedio anual del $2.5 \%$, se puede decir que las tasas de natalidad han disminuido debido a la imposición de nuevos modelos económicos en los últimos años, por lo que la subsistencia de los habitantes se ha vuelto cada vez más compleja.

\section{Migración}

El desarrollo de actividades productivas, el mejoramiento vial, la implementación de proyectos nacionales ha motivado el incremento de migrantes hacia el cantón tanto de nacional es provenientes de las provincias de: Esmeraldas, Riobamba, Bolívar, Pastaza, Chimborazo, Santo Domingo, Machala, Guayaquil, entre otras; y extranjeros procedentes de

Cuba, Colombia, Perú , USA, Europa abarcando estos últimos el $0.94 \%$ de la población total del cantón. Según estudios realizado por diferentes instituciones se ha determinado que el 
$35.45 \%$ de la población nacida en Sucre continua viviendo en la provincia; mientras que el $64.55 \%$ restante ha migrado hacia otras provincias como: Pichincha (13.5\%), Guayaquil $(8.5 \%)$, Oriente $(3.2 \%)$ entre otras ciudades (5.7\%), las edades de los individuos oscilan en un $43 \%$ entre $\operatorname{los} 21$ a 30 años, siendo motivados principalmente por la búsqueda de mejores condiciones de vida, trabajo o para la continuación de sus estudios

\section{Nivel de instrucción}

Las estadísticas presentadas por el INEC y el software REDATAM en relación a la variable de nivel de instrucción de la población del cantón Sucre se ha establecido que: el 9.53\% no cuenta con ningún nivel de instrucción, el $5.52 \%$ de la población ha realizado por los centros de alfabetización, el $1.15 \%$ obtuvo el nivel preescolar, el $31.33 \%$ tiene instrucción primaria, el $18.31 \%$ su instrucción es secundaria, el $13.25 \%$ cuenta con educación básica, el 7.5\% hasta bachillerato o educación media, el 1.26\% tiene ciclo post bachillerato, el $9.85 \%$ cuenta con instrucción superior, el $0.75 \%$ tiene post grado y el $1.55 \%$ se ignora su nivel de instrucción

\section{Profesión u ocupación}

Según datos del censo 2010 realizado por el INEC, en relación a la profesión y ocupación de los habitantes del cantón Sucre se presentan las siguientes cifras: el $29.62 \%$ son agricultores y trabajadores calificados, el $11.71 \%$ lo constituyen los trabajadores de los servicios y vendedores, el $13.51 \%$ se encuentra dedicado a ocupaciones elementales, el $9.10 \%$ lo conforman los profesionales científicos e intelectuales, el $7.95 \%$ constituyen los oficiales, operarios y artesanos, el $8.49 \%$ trabajan en apoyo administrativo y el 19.62\% restante se dedican a otras actividades.

\section{Servicios básicos disponibles}

Según los datos estadísticos del REDATAM de acuerdo al CENSO 2010 en relación a los servicios básicos de las 12.983 viviendas existentes en el cantón se ha determinado que: Agua: El 56.77\% se abastece de agua a partir de la red pública, el $28.30 \%$ de ríos, vertientes, acequia o canal, el $7.39 \%$ de pozos, el $0.22 \%$ del carro repartidor de agua y el $7.32 \%$ restante se abastece de otras formas tales como el agua de lluvia.

Servicio de Alcantarillado: El 43.17\% se encuentra conectado con la red pública de alcantarillado y el 56.83\% no cuenta con este servicio. Luz eléctrica: El 83.64\% de las viviendas del cantón cuenta con servicio eléctrico a partir de la red de la empresa eléctrico de servicio público, el $2.63 \%$ se abastece a partir de paneles solares, el $0.31 \%$ la obtiene a través de generadores de luz, el $0.19 \%$ lo adquieren de otras maneras y el $13.23 \%$ restante no dispone de este servicio. Telefonía convencional: El 23\% de las viviendas dispone de este servicio mientras que el $77 \%$ carece del mismo. Eliminación de la basura: El $63.37 \%$ lo eliminan por medio del carro recolector, el $18.87 \%$ la arrojan en los terrenos baldioso quebradas, el 7.83\% la queman, el 4.92\% la entierran, el 3.74\% la arrojan al río, acequia o canal y el $1.27 \%$ la eliminan de otras formas. 


\section{Vivienda}

El Plan de Desarrollo Cantonal 2014 menciona que el índice de crecimiento de desarrollo en vivienda es del 54.42\%.Según el censo 2010 llevado a cabo por el INEC donde sus bases estadísticas reposan en el software REDATAM se ha establecido en relación o cruce de variables al tipo de vivienda que: el $61.97 \%$ son casas o villas, el $12.34 \%$ son ranchos, el $11.11 \%$ lo constituyen los cuartos en casas de inquilinato, el 5.89\% las mediaguas, el $5.07 \%$ los departamentos ya sea encasas o edificios, el $1.88 \%$ lo conforman las chozas, el $0.70 \%$ son covachas y el $1.06 \%$ restante se encuentra constituido por otras tipologías de viviendas. Los materiales que generalmente son utilizados en la construcción de las viviendas están el zinc, hormigón (losa, cemento), palma, paja u hojas en el techo o cubierta; en relación a las paredes son de ladrillo, bloque, madera, hormigón, entre otros; en cuanto al piso pueden ser de tablas sin tratamiento previo, ladrillo, cemento o cerámica.

\section{Salud}

El proceso de colonización ha ocasionado la desvalorización y pérdida de la sabiduría ancestral de los pueblos base, en relación a la medicina tradicional. Las enfermedades más comunes en el cantón son: infecciones digestivas, parasitosis, desnutrición, infecciones respiratorias, influenza, tuberculosis, enfermedades a la piel (dermatitis, piodermitis, micosis); las cuales generalmente aquejan a los segmentos de la población más vulnerables (infantes y adultos mayores). Las causas principalmente han sido debido a los tipos de viviendas y su estado constructivo, además de evaluar su ubicación en la zona urbana o rural y el incremento de vectores que transmiten múltiples enfermedades. En relación a la infraestructura hospitalaria el cantón cuenta con centros de salud en la zona rural y dos hospitales en la cabecera cantonal, entre cinco clínicas privadas.

\section{Educación}

El Plan de Desarrollo Cantonal 2014 concierta en cuanto a los establecimientos que brindan formación académica que: A nivel primario existen 10 unidades educativas, todas ellas con jornada matutina, con un total de 752 estudiantes. A nivel medio existen siete unidades con jornada matutina, dos unidades con jornada vespertina y dos unidades con jornada nocturna, con un total de 4.227 estudiantes. En cuanto a la Educación Superior existen tres universidades, cuyas ofertas académicas se encuentran relacionadas a los campos: administrativo, contable, educación básica y media, secretariado, marketing, entre otros.

\section{Servicios Sanitarios}

Según las estadísticas del INEC en el Censo 2010 se determinó que el $43.1 \%$ de las viviendas cuenta con baterías sanitarias, el $11.75 \%$ posee pozos sépticos, el $9.5 \%$ tiene pozos ciegos, el $5.15 \%$ descargan las aguas servidas directamente a los cuerpos de agua, el $3.1 \%$ cuentan con letrinas y el $27.4 \%$ restante no cuenta con ningún tipo de servicio higiénico. De los servicios sanitarios el $62.62 \%$ son de uso exclusivo, el $14.07 \%$ lo comparten con varios hogares y el $23.29 \%$ no cuenta con servicios higiénicos.

\section{Riesgos naturales o desastre naturales.}


El Plan de Desarrollo Cantonal menciona que existe la posibilidad de un riesgo indirecto por el terremoto del 4 de agosto 1998 y abril 2016, causando daños a nivel de vivienda, perdida de vida humana, impacto en diferentes ramas productivas, empresas, negocios y locales. Existieron además afectaciones a nivel psicológico incidiendo en la emigración y en la economía de la zona, no se registran estadísticos de esto pero existió un impacto significativo en la zona.

\section{Impacto social de la imagen empresarial}

Se aplicó un segundo instrumento para medir el nivel de conocimiento y responsabilidad de gerentes y administrativos sobre la imagen comercial y su importancia en el ámbito empresarial, conformada por nueve preguntas, en la pregunta dos se le evalúa si conoce la importancia de la imagen comercial en la cual de un total de la población de 49 locales y negocios encuestados el $73.5 \%$ responde que si tiene conocimiento de la importancia y solo un 26.5 responde a que no sabe sobre la importancia que tiene una imagen para representar su negocio, tal como se muestra a continuación:

\begin{tabular}{llllll}
\hline & & $\begin{array}{l}\text { Frecuenci } \\
\text { a }\end{array}$ & $\begin{array}{l}\text { Porcentaj } \\
\mathrm{e}\end{array}$ & $\begin{array}{l}\text { Porcentaje } \\
\text { válido }\end{array}$ & $\begin{array}{l}\text { Porcentaje } \\
\text { acumulado }\end{array}$ \\
\hline Válido & SI & 36 & 73,5 & 73,5 & 73,5 \\
s & NO & 13 & 26,5 & 26,5 & 100,0 \\
& Tota & 49 & 100,0 & 100,0 & \\
& 1 & & & \\
& \multicolumn{5}{c}{ Cuadro 4 : la importancia de la imagen empresarial } \\
& Fuente: Resultados del SPSS 21.00
\end{tabular}

Se le evaluó sobre los elementos que conforman la imagen comercial para corroborar si realmente tienen conocimiento de los elementos que conforman una imagen comercial, donde se le dio opciones para que seleccionaran según su criterio o conocimiento y los resultados esperados fueron los siguientes:

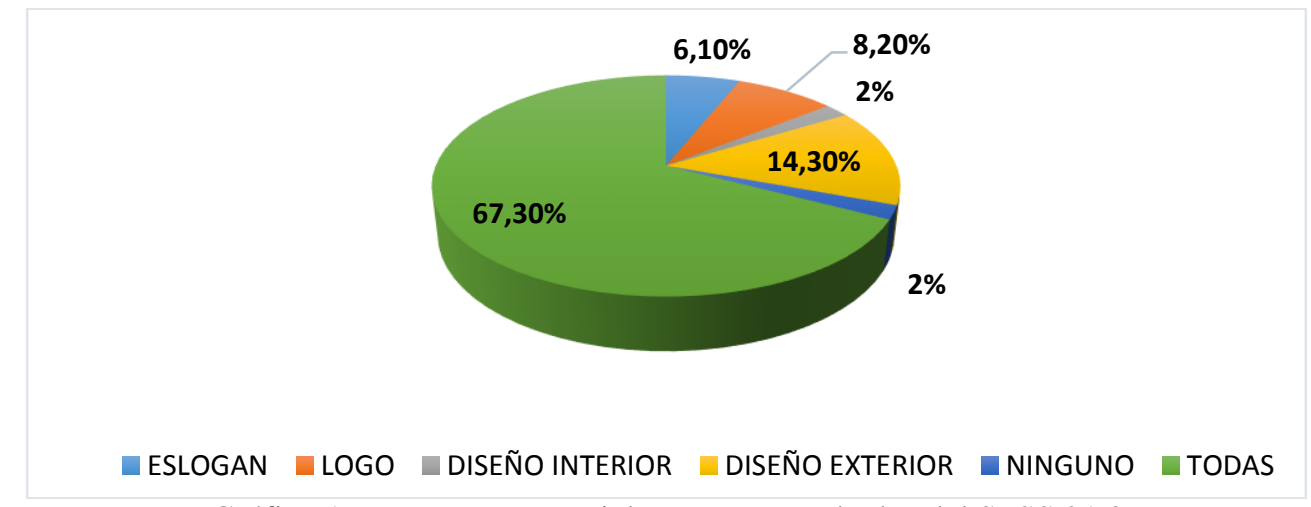

Gráfico 1: Imagen empresarial. Fuente: Resultados del SPSS 21.0

Como se observa el 67,3\% reconoce que la imagen de su negocio tiene los elementos de la imagen empresarial y solo un 37,2\% desconoce los elementos de la imagen que deben de tener sus negocios, aunque es poco representativo incide en estos negocios y en el entorno empresarial.

Se resalta que a pesar que los directivos de los negocios o locales comerciales reconocen que cuentan con una imagen comercial la cual represento $65.3 \%$ y solo un $32.7 \%$ responde no 
tener una imagen comercial, donde existe una correlación en la pregunta sobre la percepción de los clientes sobre la imagen comercial en la que responde $61.2 \%$ de buena y el $18.8 \%$ la evalúa de excelente , pero aun así un 79.6\% sugiere asistencia técnica sobre imagen comercial y solo un $18.4 \%$ no requiere de esta asistencia, por lo que los empresarios de la zona reconocen que para posicionarse mejor en el mercado y mejorar sus ventas necesitan capacitación y asistencia de profesionales en el área.

\section{Conclusiones}

Los instrumentos utilizados arrojaron resultados satisfactorio permitiendo generar estrategias de seguimiento, capacitación continua y monitoreo para medir los niveles de impacto.

El estudio de la imagen empresarial represento que el $65.3 \%$ de los locales responde afirmativamente tener una imagen empresarial de acuerdo a su razón social y solo un 32.7\% responde no tener una imagen comercial, determinando una correlación en la percepción de los clientes sobre la imagen comercial donde el $61.2 \%$ responde positivamente y el $18.8 \%$ la evalúa de excelente, pero aun así un 79.6\% sugiere asistencia técnica sobre imagen comercial y solo un $18.4 \%$.

El estudio del entorno aporto una estadística a nivel cognitivo que permitió conocer la rama de actividades de la zona y evaluar el nivel de instrucción para definir el nivel de capacitación en la zona.

Durante el estudio el 90\% de los negocios existente reduce la informalidad laboral garantizando el cumplimiento de los derechos laborales.

Durante el desarrollo del estudio el 60\% de los comerciales han definido sus estrategias de ventas y un $32.7 \%$ han manifestado su interés en rediseñar su imagen comercial.

Durante el desarrollo del proyecto el 70\% de las estrategias y técnicas aplicadas han mejorado las ventas en los negocios y locales de la parroquia, denotando que los instrumentos y materiales utilizados han sido de importancia.

\section{Recomendaciones}

1. Continuar con la aplicación de técnicas, métodos e instrumentos para medir los niveles de crecimientos de los negocios y afluencias de clientes.

2. Monitorear las actividades de capacitación continua que se realizan a través de cronograma de actividades asignado por el departamento de vinculación con la sociedad.

3. Desarrollar un software que simule las actividades del marketing empresarial que le permita desarrollar pronósticos de ventas y posicionamiento.

\section{Bibliografía}

Alcaide, J. C. (2015). Fidelización de clientes. Segunda Edición. Madrid: ESIC Editorial.

Alejandro, S. K. (2011). Marketing de fidelizacion: como obtener clientes satisfechos y leales, bajo una perspectiva Latinoamericana. Ecoe Ediciones. 
Batos, B. A. (2006). Fidelización del cliente. Introducción a la venta personal y a la dirección de ventas $1^{\text {a }}$ Edición. Vigo: Ideaspropias Editorial.

Código Orgánico De La Producción, Comercio E Inversiones, 2013.

Suplemento del Registro Oficial 351, 29-XII-2010.

Clotilde Hernández, Claudio Maubert. (2009). Fundanetos de Marketing. México: Pearson.

Chacón, E. (2009). Satisfacción y expectativa del cliente. Venezuela.

EmprendePymes. (31 de Julio de 2012). EmprendePymes. Obtenido de Matriz ANSOFF: http://www.emprendepymes.es

Esteban, I. G. (2005). Marketing de los servicios. Madrid: ESIC Editorial.

Francisco Garcia, Pedro Garcia, Mario Gil. (2009). Técnicas de servicio y atencion al cliente. Madrid: Ediciones Paraninfo.

Gosso, F. (2008). Hipersatisfacción del cliente. Primera Edición. México: Panorama Editorial.

Laura Fischer, Jorge Espejo. (2004). Mercadotecnia Tercera edición. México: McGraw-Hill.

León Schiffman, Leslie Lazar. (2010). Comportamiento del consumidor. Décima edición. Mexico: Pearson.

León, L. A. (2003). La calidad del servicio y el mejoramiento continuo en AMCCPN.

Lovelock, C. (2009). Marketin de sericios sexta edición. Mexico: Pearson.

Martha Vargas, Luzángela Aldana. (2006). Calidad y Servicio. Bogotá: Ecoe Ediciones .

Maubert, C. H.-C. (2009). Fundamentos de Marketing .Primera edición. México: Pearson.

Paz, J. C. (2010). Calidad total y recursos humanos. Salta-Argentina: Universidad Catolica de Salta.

Prieto, J. E. (2014). Gerencia del servicio (la clave para ganar todos). Tercera edición. Bogotá: Ecoe Ediciones.

Sánchez, J. M. (2007). Telemarketing. La red como Soporte de Marketing y Comunicación. Vingo: Ideaspropias Editorial .

Schnarch Kirberg, Alejandro; Schnarch Gonzáles, David. (2010). Marketing emprendedores: como identificar oportunidades y desarrollar empresas exitosas. Ecoe Ediciones . 